\title{
Una revisión de los estudios sobre diferenciación sociodemográfica, referidos a la ciudad de Madrid
}

\author{
GLoria LoRa-Tamayo D'ocon
}

Existen pocos estudios que, abarcando la ciudad de Madrid, la aborden desde el punto de vista sociodemógrafico; más numerosos son los trabajos de investigación sobre barrios, distritos o zonas concretas del municipio, donde se tratan estos temas y, por supuesto, otros donde se analiza la estructura demográfica y social de la ciudad en su conjunto, sin diferenciar áreas homogéneas específicas. En este artículo hacemos una revisión de aquellos trabajos que consideramos de interés para el conocimiento de la distribución espacial de la población de la ciudad de Madrid, según su estructura social y demográfica, tema que en los últimos años ha merecido mayor atención por parte de geógrafos, sociólogos y estudiosos de Madrid.

\section{ESTUDIOS GENERALES}

Uno de los estudios más interesantes que, por su antigüedad, tiene casi un valor histórico, pero cuya utilidad es innegable, es el Informe Sociológico sobre la situación Social de Madrid de 1967, patrocinado por la Fundación Foessa. En él se realiza una división de la ciudad en Áreas Históricas, Distritos y Zonas, hallándose diferencias sociodemográficas fundamentales en los tres niveles, en base al análisis de varias características de la población residente en ellas: evolución de la misma, sexo y edad, estado civil, estructura ocupacional, la familia, la alimentación, la 
salud, la vivienda, niveles de instrucción, trabajo, remuneración, equipamiento y problemas de la comunidad.

La diferenciaciones espaciales más acusadas se producen, en todos los temas analizados, entre las tres unidades históricas y, sobre todo, entre el Madrid Interior (Centro y Ensanche) y la Periferia - dos «madriles" distintos y contrapuestos-, contando el primero con proporciones mayores de población madrileña, clases sociales más acomodadas, familias más pequeñas, mayor proporción de población adulta y "vieja", también proporciones más elevadas de población activa, menos trabajadores manuales, más directivos y profesionales liberales y un nivel de instrucción más elevado; frente a la Periferia, área fundamentalmente heterogénea y en muchos casos "poco urbana", que en ese momento estaba sufriendo un proceso de crecimiento acelerado, en base a una inmigración masiva, compuesta sobre todo por población venida del medio rural y cuyas características fundamentales podian resumirse asi: población joven, compuesta por matrimonios recientes con hijos pequeños, poca cualificación y bajo nivel de instrucción, temprano acceso al mundo laboral y elevada proporción de trabajadores manuales. Asimismo, existían diferencias notables entre Centro y Ensanche, considerándose esta última área como la más rica de Madrid y la primera como la de población más envejecida.

La multitud de variables analizadas a lo largo del Informe, se resumía en tres, consideradas como las más indicativas a la hora de diferenciar áreas homogéneas en la ciudad: el nivel de posición social (índice formado a partir de las variables: ocupación de la población masculina, estudios del marido, estudios de la mujer, suma de ingresos familiares y clase social subjetiva), la juventud de la población (índice: menores de 15 años -mayores de 66) y el crecimiento de la zona desde 1950.

Las puntuaciones más elevadas para las variables que configuran el nivel de posición social, se conseguían siempre en las zonas que componen el Centro y Ensanche, según esta clasificación. Dentro de la Periferia, el nivel de ingresos más alto lo tenía Carabanchel, seguido de Ventas, Moratalaz, Canillas-Hortaleza y Tetúan; también Tetúan, Ventas y Canillas-Hortaleza (por este orden), se hallaban a la cabeza de la Periferia en las puntuaciones de ocupación de los varones; Moratalaz, Villaverde,Tetúan y Ventas, en estudios del marido; Moratalaz, Tetuan, Carabanchel y Ventas en estudios de la esposa. En todas estas variables, Villaverde y Vallecas contaban casi siempre con las puntuaciones más bajas de todo el municipio, constituyendo los "verdaderos proletariados". 
En relación con la juventud de la población y dentro de este mismo nivel zonal, Canillas-Hortaleza figuraba con la más alta puntuación, seguido de Villaverde, Ventas, Carabanchel y Vallecas. Las zonas del Centro y Ensanche tenían los mismos puestos. Lo mismo ocurría con el crecimiento intercensal, donde Villaverde ocupaba la primera posición, seguido de Moratalaz, Canillas-Hortaleza, Ventas y Vallecas.

Como conclusión, se consideraba el municipio de Madrid claramente estructurado en cuatro grandes estratos sociales "bastante bien definidos y homogéneos": un Madrid acomodado, un Madrid medio, el Madrid pobre y el Madrid indigente. Su distribución espacial queda recogida en la figura 1.

A. Huetz de Lemps, en 1972, establece una diferenciación entre el Centro histórico de Madrid -de población envejecida, donde residen clases populares y medias-, el Ensanche - donde se combinan clases populares, medias, acomodadas, burguesas y aristocráticas- y la Periferia, el área más compleja, donde el autor diferencia "los viejos barrios populares", residencia de clases medio-bajas; "barrios pobres posteriores a la guerra civil», donde se integran los poblados de chabolas, "poblados de absorción" y "poblados dirigidos", todos ellos lugar de residencia de las clases sociales más bajas; "los barrios de clase media"; los de "clase alta", dentro de los que diferencia los constituidos por grandes edificios de apartamentos y los de viviendas unifamiliares con jardín; $y$, por último, los «pueblos vecinos absorbidos". El trabajo de Huetz de Lemps, fundamentalmente descriptivo, es un estudio de tipo general, cuyo enfoque no analiza específicamente la diferenciación residencial de la ciudad, sino que cubre otros aspectos morfológicos y funcionales; de ahi su simplificación de las categorias sociales y su metodología puramente intuitiva, en la asignación de los tipos. Su valor, sin embargo, es indicativo.

También tiene un valor indicativo el estudio que F. Chueca Goitia (1974) realiza sobre Madrid, enfocado desde el punto de vista de la Ecologia Social. Goitia acopla la ciudad de Madrid a un «esquema mixto concéntrico-sectorial», en el cual la parte Norte está constituida socialmente por zonas de expansión radiales - sectores-y la parte Sur por zonas concéntricas, discurriendo el "eje social divisorio" que las separa, por las calles de Segovia, Colegiata, Magdalena, Moratín y Espalter, y cortando un trozo del Retiro. Al sur de éste, diferencia cuatro zonas concéntricas, correspondientes al Madrid de clase baja, la más exterior de las cuales es el área de residencia de los estratos sociales inferiores de la ciudad, "y en última consecuencia, el refugio de mendigos, inadaptados, hampones y delincuentes vulgares". Al norte del eje, el esquema es 


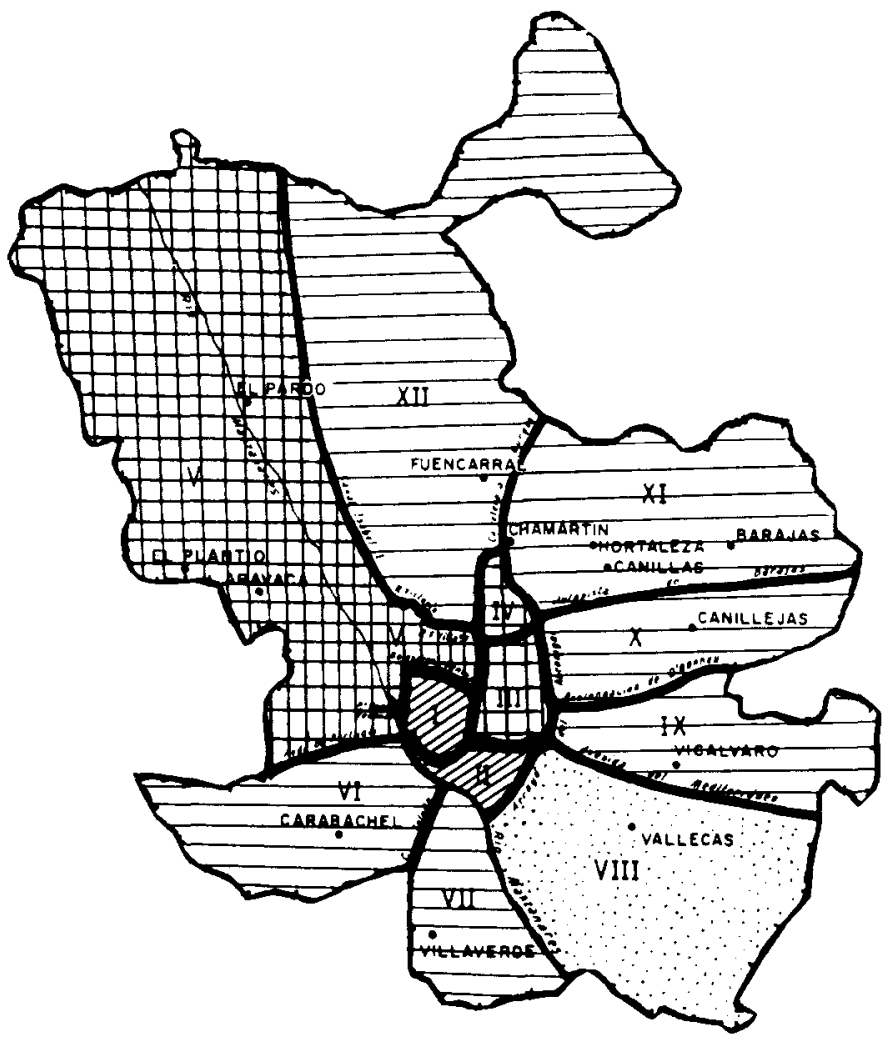

1. Centro.

II. Legazpi.

III. Salamanca.

IV. Chamartín.

V. Chamberi-Argüelles.

VI. Carabanchel.

VII. Villaverde.

VIII. Vallecas.

IX. Moratalaz.

$X$. Ventas.

XI. Canillas-Hortaleza.

XII. Tetuan-Fuencarral.

\begin{tabular}{|c|c|c|c|}
\hline Desur & roilo historico & & División en zonas \\
\hline EL & Centro. & & Centro. \\
\hline $\mathbf{E L}_{\mathbf{L}}$ & ENSANCHE. & 2. & Legazpi. \\
\hline ". & $"$ & 3. & $\begin{array}{l}\text { Salamanca. } \\
\text { Chamartin. }\end{array}$ \\
\hline$"$ & $"$ & 5. & $\begin{array}{l}\text { Chamartın. } \\
\text { Chamberi-Argüelles. }\end{array}$ \\
\hline $\mathrm{L}_{\| A}$ & Perifenta. & 6. & Carabanchel. \\
\hline 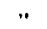 & .. & 8. & vallecas. \\
\hline$"$ & " & 9. & Moratalaz. \\
\hline$"$ & " & 10. & Ventas. \\
\hline 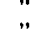 & " & 11. & Canillas-Hortaleza. \\
\hline$"$ & & 12. & Tetuan-Fuencarral. \\
\hline
\end{tabular}

\section{Madrid Acomodado \\ $E$ iladrid Pobre \\ WIIlA Madrid lledio \\ $\therefore \therefore$ Madrid Indigente}

Fig. 1. División en zonas de la ciudad de Madrid, según el informe Foessa (1967). (Elaboración propia a partir de los datos del Informe Foessa). 
radial por sectores, sucediéndose de oeste a este una cuña verde (Casa de Campo), un sector de clase alta (Argüelles y Ciudad Universitaria), un sector de clase media (desde Princesa hasta Almagro, aproximadamente), un sector de clase alta (cuyo eje es la Castellana) y un último sector, donde se combina un área de clase alta hacia el centro y de clase media en la porción distal. En la periferia de estos sectores se forman los suburbios, cuyas características residenciales obedecen a la ley «a sector medio corresponde suburbio medio-bajo y a sector alto corresponde suburbio medio-alto".

Compartimos la opinión de B. C. Jiménez Blasco (1986: 285 y ss.) sobre este esquema, que resulta interesante, pero intuitivo, en nuestro criterio, además al modelo se basa en el Madrid de los años 40-50 (a juzgar por algunas descripciones y por los datos de población que aporta, los más recientes de los cuales son de 1940), a pesar de corresponder la publicación a los años 70 , precisamente, en estos suburbios que según el autor "son zona de fermentación de Madrid, donde pueden presentarse imprevistas estructuras, novedades en lo físico y en lo social», es donde más cambios morfológicos y sociales se han producido en las últimas décadas.

Como resumen a los planteamientos generales sobre la diferenciación social de Madrid, la oficina Municipal del Plan (1982) elabora una serie de mapas que «muestran la dicotomía de la estructura urbana, que produce un Madrid bipolar», donde trazando una línea que enlazara la carretera de Barcelona (N. II) con la de Extremadura (N. V), al Norte de ella, sobre un espacio mayor, asiento del 48 por 100 de la población de la ciudad, residirían el 82 por 100 de los titulados superiores y únicamente el 32 por 100 de los trabajadores manuales. La localización preferente de estos últimos seria al sur de ésta línea, precisamente donde el nivel de dotaciones es peor (el 16 por 100 del empleo terciario, el 20 por 100 de las instalaciones deportivas y el 18 por 100 de las plazas de BUP, para el 52 por 100 de la población de la ciudad).

Efectivamente, los estudios de B. C. Jiménez Blasco (1986) sobre la segregación ocupacional en la ciudad de Madrid, demuestra cómo es precisamente la categoria de los trabajadores manuales la que adopta localizaciones más especificas dentro de la ciudad, siendo los distritos con mayor concentración de ellos, en orden decreciente, Vallecas, Carabanchel, Latina y Villaverde, y hallándose poco representados en los distritos correspondientes a la "almendra". Por su parte, las máximas concentraciones de los profesionales de los cuadros superiores (el polo opuesto de la escala social), se encuentran en el Ensanche (excepto en 
su zona meridional), barrio de Cuatro Caminos y distrito de Chamartín, según la autora.

Los mapas antes mencionados sobre la polarización de las clases sociales de Madrid, que se encuentran en el documento "Recuperar Madrid", tienen su base en otros que, a su vez se presentan en los "Documentos Sectoriales" de la Oficina Municipal del Plan (1983) y que siven de base para la estimación de los déficit en el equipamiento de la ciudad.

La cartografía se realiza en base a tres unidades de análisis: de mayor a menor, áreas, zonas y barrios municipales. Estas unidades se clasifican según tres tipos de niveles socioeconómicos: alto, medio y bajo. La estratificación se efectúa sobre el "valor promedio de las variables indicativas de la clase social de las familias residentes en cada barrio: porcentaje de población analfabeta o con estudios primarios incompletos y porcentaje de trabajadores manuales". El indicador utilizado es parecido al empleado en el Informe Foessa, si bien, al ser las unidades de análisis más pequeñas y la fecha más actual, esta aproximación cobra nuevo interés. Desconocemos, sin embargo, los criterios de delimitación de los umbrales o líneas divisorias entre cada tipo de status socioeconómico. Por otra parte, la propia diferenciación en tres únicos tipos de nivel socieconómico, resulta bastante simplista.

Las áreas que según la Oficina Municipal del Plan, tienen un nivel socieconómico alto se sitúan fundamentalmente dentro de la "almendra madrileña", abarcando los distritos de Retiro, Salamanca, Chamartín y Chamberí, y prolongándose al NW en parte de los de Moncloa y Fuencarral, y al NE, en el sector norte del de Ciudad Lineal. Las áreas de clase baja ocupan una orla periférica al núcleo central, localizándose, sobre todo, al SE de la ciudad, cubriendo la totalidad de los distritos de Mediodía y Vallecas y parte de los de Moratalaz y San Blas. Las áreas restantes son de nivel medio; ocupan mayoritariamente la Periferia Norte del municipio y los distritos del Centro, Arganzuela, Latina y parte de los de Carabanchel, Moratalaz y Ciudad Lineal. A nivel zonal, la distribución espacial de las clases socioeconómicas es parecida. Las diferencias de la distribución espacial de las clases sociales a nivel barrio, quedan reflejadas en la figura 2.

Por último, no queremos dejar de mencionar algunos estudios sobre la diferenciación espacial de Madrid, abordada únicamente desde el punto de vista de la estructura demográfica.

Entre los más antiguos figura el de A. Abellán (1976) que, partiendo de los actos del Censo de 1970, diferencia dos tipos de distritos: los 
Una revisión de los estudios sobre diferenciación sociodemográfica, ...

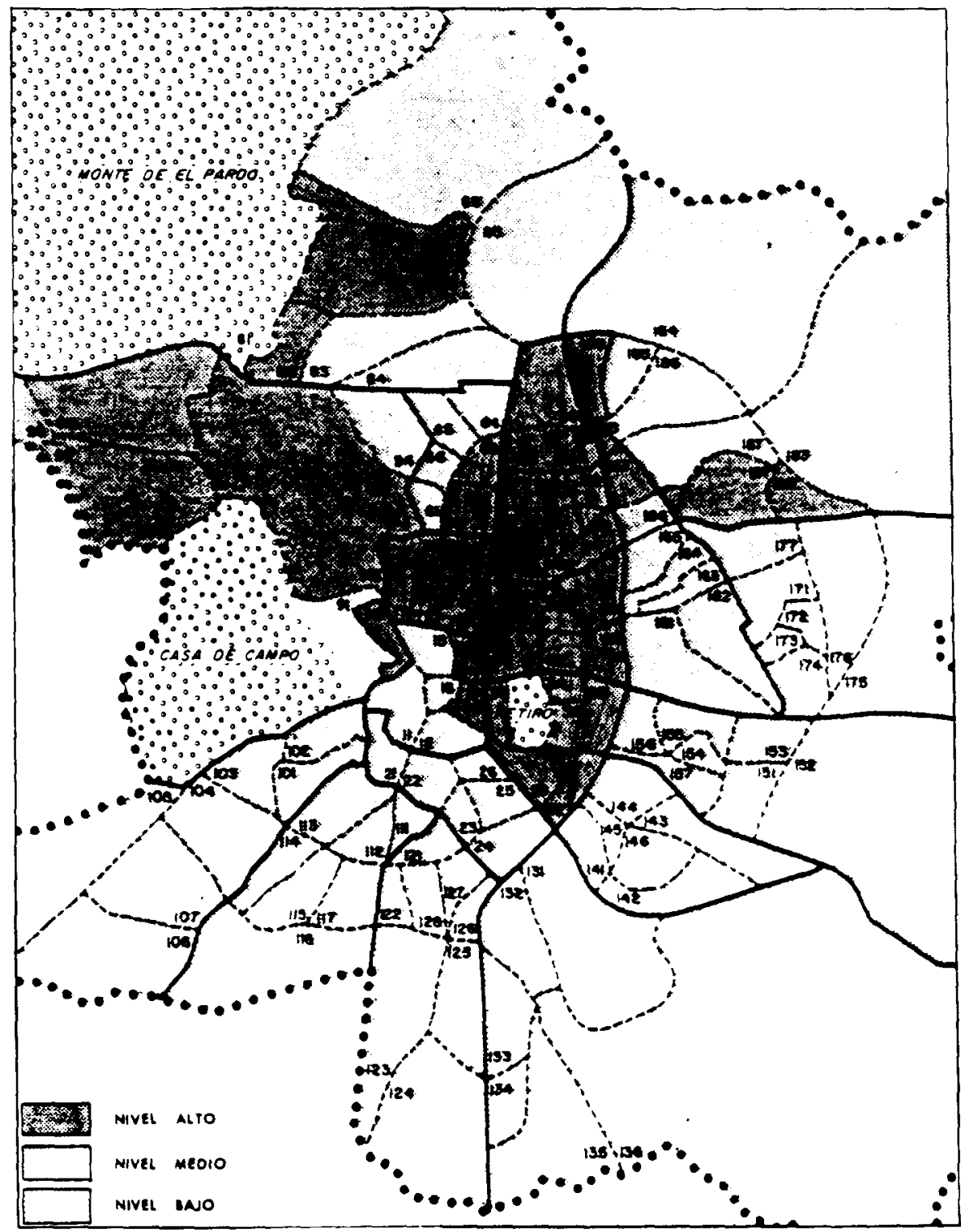

Fig. 2. Nivel socioeconómico de los barrios de Municipio de Madrid, según la Oficina Municipal del Plan (1983). ("Documentos sectoriales» de la Oficina Municipal del Plan. Ayuntamiento de Madrid. 1983). 
envejecidos (que cuentan con una proporción de entre el 15 y el 25 por 100 de población menor de 14 años y entre un 10 y un 18 por 100 de población de más de 65), como Centro, Universidad, Chamberi, Buenavista, Retiro, Mediodía y Arganzuela; y los jóvenes (con más del 30 por 100 de población menor de 14 años y menos del 7 por 100 de mayores de 65), como Tetuán, Chamartín, Ventas, Villaverde, Carabanchel y Vallecas. En líneas generales, los distritos del Centro se caracterizan por un tipo de pirámide cuyo escalón inferior es muy reducido, "con un típico proceso "hongo"", mientras las correspondientes a los distritos de la Periferia, tienen bases extraordinariamente amplias.

En esta misma línea de análisis, B. C. Jiménez Blasco (1986) elabora las pirámides de los 18 distritos actuales de Madrid, según los datos del Padrón Municipal de Habitantes de 1981, estableciendo tres tipos básicos: 1) distritos jóvenes (Ciudad Lineal, Fuencarral, Hortaleza, Mediodía, Moratalaz, San Blas y Villaverde), 2) distritos intermedios (Carabanchel, Chamartín, Latina, Moncloa, Tetuán y Vallecas) y 3) distritos envejecidos (Arganzuela, Centro, Chamberí, Retiro y Salamanca). Asimismo, clasifica las pirámides según el equilibrio que guardan las barras correspondientes a los distintos grupos de edad en: distritos compensados (Arganzuela, Carabanchel, Ciudad Lineal, Chamartín, Latina, Mediodia, Moncloa, Retiro y Salamanca) y distritos con fuertes discontinuidades entre los escalones de sus pirámides (los restantes). En cuanto a la composición por sexo, diferencia los distritos con un predominio de mujeres (Arganzuela, Carabanchel, Centro, Chamberí, Chamartín, Moncloa, Retiro y Salamanca), bastante coincidentes con los más envejecidos; distritos con un equilibrio entre sexos (Ciudad Lineal, Hortaleza, Latina, Mediodía, San Blas, Tetuán, Vallecas y Villaverde) y los distritos con un predominio de varones (Fuencarral y Tetuán).

Con base en los datos del Padrón Municipal de Habitantes de 1986, exiten dos estudios interesantes sobre la distribución espacial de la población de Madrid según su estructura demográfica, a un nivel de desagregación mayor, ésto es, tomando como unidad de análisis el barrio municipal.

A. García Ballesteros et alli (1989) reflejan los contrastes en la distribución de los grupos demográficos empleando tres indicadores básicos: las desviaciones con respecto a la pirámide-tipo de Madrid (de donde se deduce una tipología de siete grupos de estructuras, en función del perfil de las pirámides), la edad mediana de la población y el porcentaje de población anciana. Los barrios más envejecidos se localizan en el Distrito Centro y gran parte de los barrios del Ensanche del siglo XIX, pertenecientes a los distritos de Chamberí, Salamanca, Retiro, Moncloa y Argan- 
zuela, y, en menor intensidad, los situados en la periferia del Ensanche y algunos de Extrarradio y Periferia próxima, localizados a lo largo de las carreteras de Extremadura, Aragón, Valencia y Andalucía-Toledo. En cuanto a los barrios más jóvenes, se localizan en los distritos periféricos de Hortaleza, Vallecas y Fuencarral y secundariamente en San Blas, Ciudad Lineal y Latina. Con un segundo nivel de intensidad en relación con la juventud de la población, aparecen algunos barrios de los distritos de Villaverde, Moratalaz y Vallecas.

El análisis de la edad mediana de la población señala igualmente el contraste existente entre los barrios del centro de la ciudad, que superan los 43 años y los que conforman el borde exterior del municipio, con valores inferiores a 32 años. El contraste se puede sintetizar en la existencia en el centro de Madrid de algunos barrios con proporciones superiores al 23 por 100 de población anciana, frente a otros que no superar el 11 por 100 , localizados en la periferia.

Esta distribución espacial, clásica, por otra parte, de otras ciudades desarrolladas de Europa Occidental, queda asimismo destacada en el estudio realizado por M. J. Aguilera y P. Gonzalez Yanci (1989), que utilizan como fuente igualmente el Padrón Municipal de Habitantes de 1986, expresándose la estructura espacial en «un centro envejecido en torno al que se suceden aureolas de barrios cuyos niveles de enevejecimiento van decreciendo" (1989: 75) -aunque, por supuesto, existen excepciones cuya causa fundamental es el trazado de las principales vías de comunicación-. La correlación entre el porcentaje de ancianos y la distancia al centro urbano en la ciudad de Madrid, por otra parte, queda expresada en los valores $-0,65$ y $-0,75$ del coeficiente de correlación de Pearson y el indice de correlación de Spearman, respectivamente, calculados por las autoras.

\section{ESTUDIOS MULTIVARIANTES RECIENTES}

Existen toda una serie de estudios de gran interés, que abordan el tema de la diferenciación residencial de Madrid empleando un número mayor de indicadores y utilizando técnicas de análisis multivariante. Comentaremos a continuación los estudios realizados por $M$. Castells (1981), A. del Campo (1983) y B. C. Jiménez Blasco (1984 y 1986), todos ellos dentro de esta línea de investigación, si bién, resulta interesante, antes de iniciar su revisión, recoger aquí el Modelo de Áreas Sociales aplicado a Madrid por esta última autora (1986). 


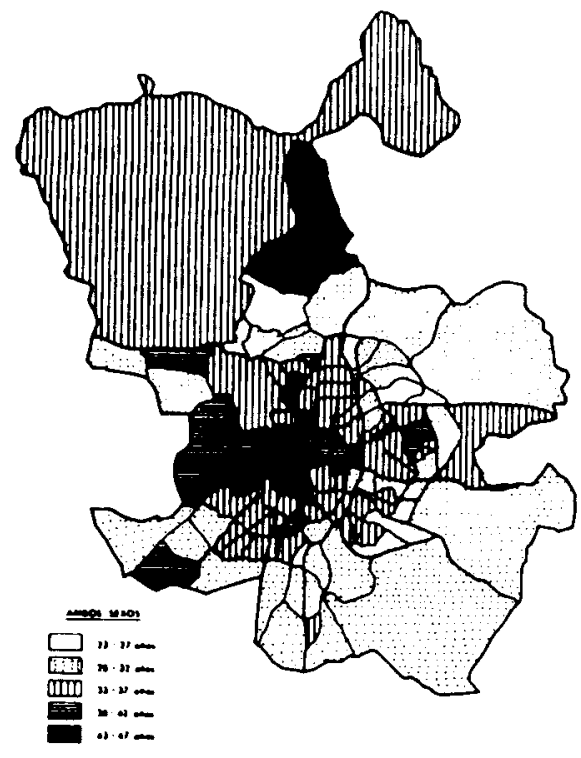

Fig. 3. Edad mediana de los barrios del municipio de Madrid, según A. García Ballesteros et alli (1986).

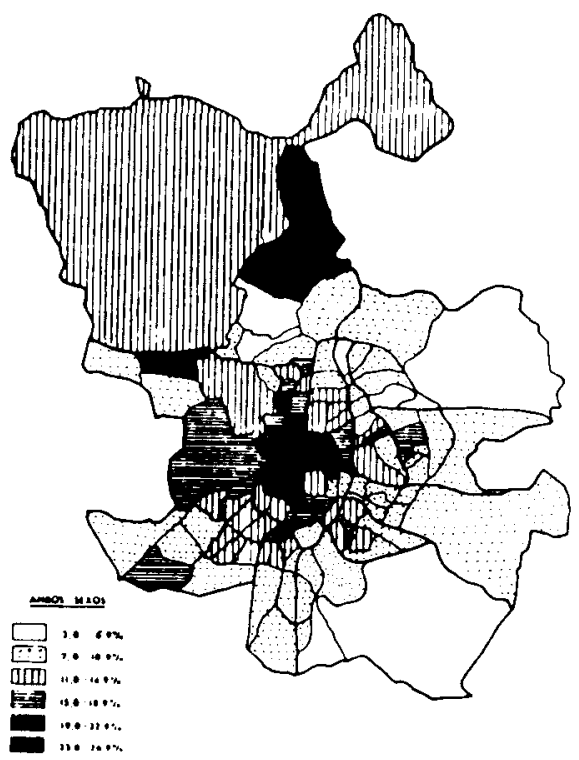

Fig. 4. Porcentaje de mayores 65 años sobre el total de la población en los barrios de Madrid, según A. García Ballesteros et alli (1986). 
La Teoría de Análisis de Áreas Sociales y el modelo de ella derivado, deben encuadrarse dentro de un enfoque macrosocial, donde las "agragaciones urbanas son analizadas, no como fenómenos únicos con sus propios principios de organización, sino como partes de una más amplia sociedad moderna" (Timms, D., 1965: 253). Su sistematización se debe a E. Shevky y W. Bell, (1955), quienes, partiendo de una teoria general sobre los postulados característicos de la sociedad industrial (cambios en el grado e intensidad de las relaciones, diferenciación de funciones y complejidad de organización), entendidos como aspectos de una escala creciente, derivan de ellos unas tendencias (cambios en la distribución de los oficios, cambios en la estructura de la actividad productiva y cambios en la composición de la población), que, a su vez, provocan otra serie de cambios en la organización de un sistema social dado (cambios en el sistema de las ocupaciones, basados en la función, cambios en los modos de vida -introducción de las mujeres en las ocupaciones urbanas y extensión de patrones familiares alternativos- y redistribución de la población en el espacio -aislamiento y segregación de grupos-), concluyendo en la selección de "tres reflexiones estructurales de cambio que pueden ser utilizadas como factores para el estudio de la diferenciación y estratificación, en un momento determinado, en la sociedad moderna". Estos factores fundamentales o ejes básicos son: Rango Social o Status Social, Urbanización o Status Familiar y Segregación o Status Étnico.

El primero de estos ejes hace referencia al papel fundamental que la ocupación de cada individuo tiene en su estimación social, su nivel económico y su grado de instrucción, dentro de la sociedad moderna.

El Status Familiar hace referencia al distinto papel que cada individuo de una familia tiene dentro de la economía moderna y sus consecuencias: reducción del tamaño de la familia, entrada de la mujer en la actividad productiva y cambios en los estilos y forma de vida; contrapone el "familismo", definido como la preferencia por el patrimonio y los hijos, frente a alternativas tales como la promoción profesional y el consumo.

El último eje diferenciador, la Segregación o Status Étnico, es una consecuencia de la movilidad y la inmigración, que provoca el aislamiento de grupos minoritarios.

Para la medida de estos ejes o dimensiones básicas de diferenciación social, los autores seleccionan una serie de indicadores, de donde se deducen unos índices. El primero de ellos, Rango Social o Status Económico, adquiere un valor numérico determinado por los indicadores Ocupación (proporción de obreros manuales), Educación (proporción de mayores de 25 años en niveles de instrucción bajos) y Renta. El se- 
gundo, Urbanización o Status Familiar, adquiere también un valor numérico a partir de la Tasa de Fertilidad, la Tasa de Mujeres Activas y la proporción de Viviendas Unifamiliares. El último índice, Segregación o Status Étnico, se deduce del porcentaje "negros", "otras razas" o "extranjeros blancos", sobre la población.

Los valores de los dos primeros índices que llevan sobre unos ejes de ordenadas, divididos en cuatro intervalos que se corresponden con los cuartiles del rango de las puntuaciones en estos índices y que configuran un rectángulo subdividido en 16 porciones que resumen los 16 tipos básicos de espacios sociales considerados por Shevky y Bell. Sobre este esquema se monta el indice de segración - que ha mostrado ser poco útil en los países donde no existen minorías étnicas-, dividiendo cada espacio en dos, según el valor del índice sea alto o bajo.

A pesar de las críticas que ha tenido la Teoria y el Modelo de las Áreas Sociales, nos parece interesante su aplicación a Madrid, llevada a cabo por B. C. Jiménez Blasco (1986), en base a los datos de la Rectificación (a 1977) del Padrón Municipal de Habitantes de 1975, a nivel de barrio municipal, cuyo resultado se refleja en las figuras 5,6 y 7 .

En la primera se cartografía el índice de Rango Social, calculado (por falta de datos) en base a unos indicadores opuestos a los establecidos por Shevky y Bell: profesionales en cuadros superiores y titulados superiores. El primer indicador no resulta demasiado adecuado en la significación de los grupos de status social más elevado, ya que, como la autora precisa, no incluye todas las ocupaciones de rango social elevado; asimismo, el volúmen de titulados superiores en Madrid es tan reducido en relación con el volumen de población, que los valores del índice son muy bajos. Por otra parte, los grupos sociales más bajos adoptan en la ciudad distribuciones especiales más segregadas (como la autora comprueba en otra parte del mismo trabajo), por lo que la adopción de los indicadores establecidos por Shevky y Bell habría resultado mucho más significativa de la diferenciación especial en el índice de rango social en el municipio de Madrid. Con todo, el Mapa 5 refleja el contraste Periferia (con los valores más bajos, a excepción de los barrios que bordean la carretera de la Coruña) - Zona interior. Dentro de esta última, los barrios del Ensanche Norte y Este presentan los valores más elevados. En cuanto a la Periferia, los niveles inferiores corresponden a la totalidad de los barrios integrados en los distritos meridionales de Latina, Carabanchel, Villaverde, Mediodía, Vallecas y Moratalaz.

La figura 6 refleja los contrastes entre barrios según cuatro tipos o grados de «urbanización». No existe ningún barrio localizado en el inter- 


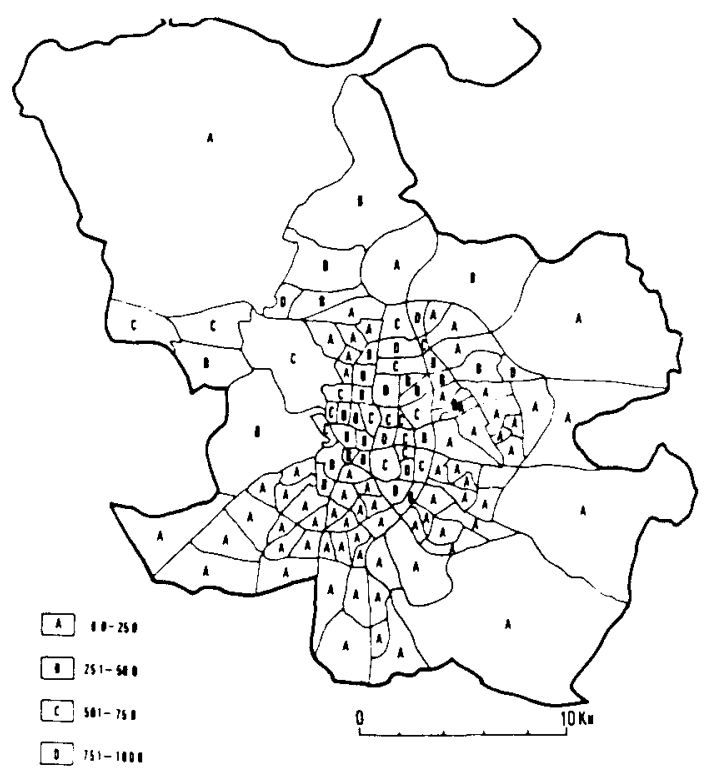

Fig. 5. Indice de rango social según B. C. Jiménez Blasco (1986).

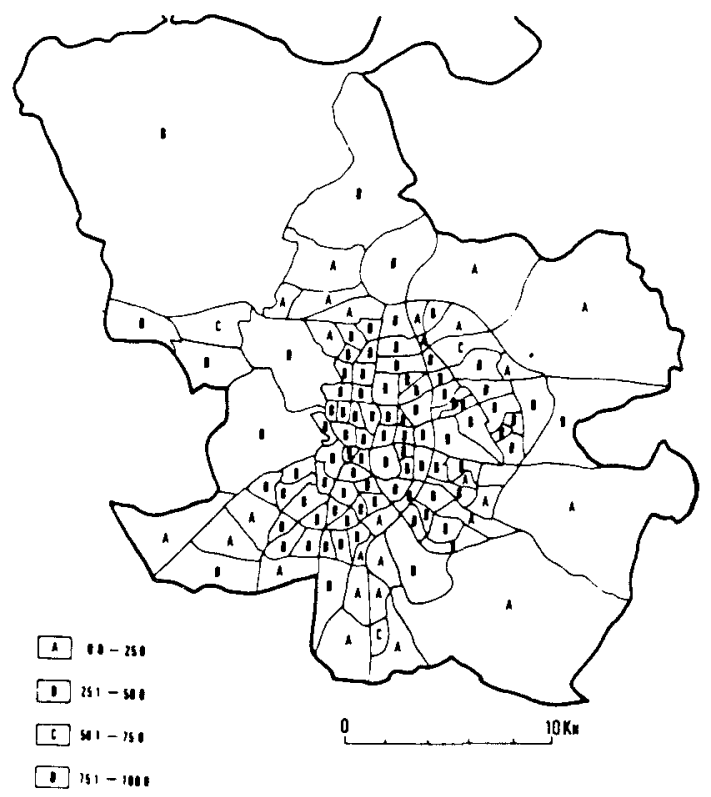

Fig. 6. Indice de urbanización, segun B. C. Jiménez Blacos (1986). 
valo superior, encuadrándose la mayor parte de ellos dentro de los dos primeros intervalos, si bien los niveles más bajos se dan en barrios periféricos. Ello significa un predominio del grado de urbanización bajo o una tendencia al "familismo" en la ciudad de Madrid. Para B.B. Jiménez Blasco, los dos indicadores del segundo índice del modelo no son adecuados para medir el grado de urbanización de Madrid, aunque lo puedan ser para las ciudades americanas. En nuestro criterio, es seguramente el desfase de los datos lo que dá esta estructura, ya que en los últimos 10 ó 15 años los cambios sociales acaecidos en el país y en la ciudad de Madrid, en relación con la estructura familiar, incorporación de la mujer al mundo laboral y descenso de la tasa de fertilidad, han sido acelerados, por lo que un nuevo ensayo de cartografía del índice para Madrid, a partir de los datos del Padrón de 1986, posiblemente demostrará un retroceso del "familismo" y, por tanto, un aumento de los niveles de «urbanización».

El último mapa refleja los resultados del modelo aplicado a Madrid, aunque sin incluir el índice de Segregación, por no considerarlo aplicable a nuestra ciudad. A pesar de las autocríticas de la autora a su análisis, basadas sobre todo en la escasa adecuación al modelo del índice de rango social por ella empleado, el modelo «proporciona una imagen bastante acertada de las diferentes zonas de Madrid: 1) un centro-norte más urbanizado y con medio-alto rango social; 2) un centro-sur urbanizado, pero con menor rango social; 3) una periferia norte-oeste, con unos índices de urbanización mayores que los reales y con un rango social alto y 4) una periferia sur-este con escasa urbanización y bajo rango social»». (1986: 436).

Estamos de acuerdo en las críticas que la autora hace a esta aplicación a Madrid del Modelo de Áreas Sociales, consecuencia de la falta de datos disponibles para uno de los indicadores. Consideramos, por otra parte, que un nuevo ensayo empleando los indicadores precisos señalados por Shevky y Bell y en base a los datos de 1986, a pesar de que la técnica de la Áreas Sociales esté hoy en día desfasada, podría resultar de interés.

De esta manera enlazamos con la revisión de los trabajos que apuntamos anteriormente, que realizan un estudio fundamentalmente sociodemográfico de la ciudad de Madrid, utilizando técnicas de análisis multivariante $-\mathrm{y}$, por tanto, manejando un número crecido de indicadores- entre las cuales las más común es el Análisis Factorial.

El empleo de las técnicas de Análisis Factorial en el estudio de la diferenciación residencial y en el Análisis de Áreas Sociales, viene a 


\section{TIPOLOGIA \\ $D E$ \\ AREAS SOCIALES}

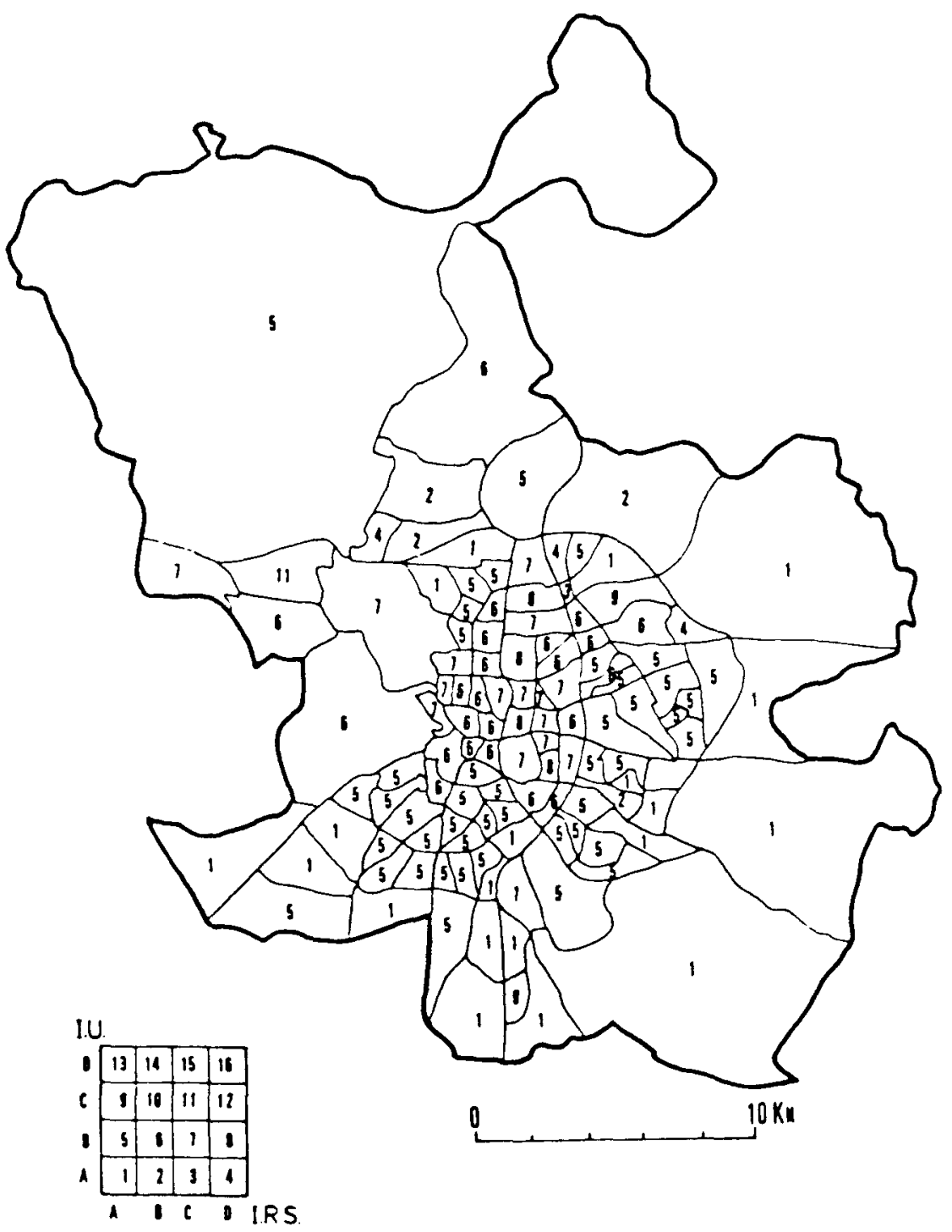

Fig. 7. Modelo de áreas sociales aplicado a Madrid por B. C. Jiménez Blasco (1986). 
paliar una de las deficiencias más importantes de esta última técnica analítica: el apriorismo en la elección de los ejes diferenciadores y de los indicadores que componen cada eje. El Análisis Factorial es una técnica inductiva que permite la identificación del orden subyacente en una serie de datos, reduciendo un número considerable de variables correlacionadas a unos cuantos factores básicos, suficientes por sí mismos para dar cuenta de prácticamente toda la variación observada en los fenómenos estudiados. La aplicación de estas técnicas «a los datos que describen la diferenciación residencial de la población, generalmente de la población urbana» (Timms, D. 1976: 100), se denomina Ecología Factorial.

Los análisis de Ecología Factorial aplicados a ciudades han respaldado, en términos generales, la hipótesis del modelo de Áreas Sociales, flexibilizándolo y enriqueciéndolo: se han evidenciado nuevos factores o ejes diferenciadores; se ha puesto de manifiesto la distinta importancia de los diferentes factores según la localización de las ciudades en unas coordenadas económicas, étnicas y culturales; por otra parte, los indicadores o variables de más peso dentro de cada factor, puede variar del rígido esquema de Áreas Sociales y también se encuentran en algunas ciudades correlaciones entre los distintos factores.

En conjunto, en los estudios realizados hasta ahora, los dos factores clásicos de diferenciación sociodemográfica son:

1. El Status Socioeconómico o Rango Social, factor que suele explicar la mayor parte de la varianza, agrupando variables relacionadas con los trabajadores profesionales y directivos, altos niveles de educación y renta y vivienda de calidad superior a la media, con cargas positivas y los trabajadores manuales, bajo nivel de intrucción y viviendas de baja calidad, con cargas negativas.

2. El Status Familiar, que agrupa variables de tipo demográfico: número de personas por familia, fertilidad, mujeres solteras o viudas, etcétera... "Las poblaciones caracterizadas por un alto valor de este factor, son aquellas con elevado porcentaje de población infantil, pocos ancianos y pocos adultos solteros, predominio de vivienda unifamiliar, áreas alejadas de la zona más antigua de la ciudad..." (Timms, D. 1976: 107).

Dentro de las técnicas de análisis factorial se encuadra el estudio de diferenciación social y funcional del espacio madrileño realizado por $M$. Castells (1981). Se trata de un doble estudio de análisis factorial "a ciegas", aplicado, por una parte, a 68 variables sociodemográficas de los 120 barrios de la capital (en base a los datos de población de la Rectificación a 1977 del Padrón Municipal de Habitantes de 1975) y por otra, a 50 variables "urbanas", de las 62 zonas de análisis en que COPLACO 
divide el municipio (datos extraidos del «Banco de Datos" de COPLACO). Los dos estudios son independientes - lo cual resta interés al trabajo- y no son un fin en sí mismos, sino que constituyen un paso para relacionar las características sociales y urbanas de las distintas áreas de Madrid con el movimiento social urbano, tema central del estudio. Por ello, los pasos seguidos en el proceso de análisis factorial no están suficientemente explicitados, las variables introducidas son demasiado numerosas y los resultados obtenidos son confusos en algunos casos, por lo que no deben entenderse como totalmente rigurosos, a nuestro juicio.

Del primer análisis se deducen cuatro factores de diferenciación sociodemográfica: 1) Nivel Social, 2) Barrio de Inmigración Reciente, 3) Envejecimiento Social y 4) Actividad Burocrática. De ellos, los más claros son el primero y el tercero.

El eje diferenciador Nivel Social agrupa con cargas positivas las variables indicativas de nivel de instrucción alto y medio-alto y los cuadros superiores, profesionales y actividades de gestión de empresas, y con cargas negativas la población obrera en general y las variables indicativas de bajo nivel de instrucción. En su representación cartográfica (Fig. 8) se observa cómo los barrios de más alto nivel social (en tramas oscuras) se localizan fundamentalmente dentro de la "almendra" madrileña y sobre todo en el Ensanche: así, del Distrito de Salamanca, todos los barrios, a excepción de Castellana, se sitúan en el umbral superior, y del de Chamartín, todos menos Castilla dentro de los dos umbrales más altos; el Distrito de Chamberí cuenta con dos barrios dentro del primer umbral y otros tres en el segundo. La Periferia también tiene barrios de alto nivel social: dentro del primer umbral se sitúan Peñagrande -en Fuencarral-, Colina, Atalaya y Costillares -en Ciudad Lineal- y Valdefuentes y Paloma -en Hortaleza- En segundo umbral, se sitúan Fuentelarreina -en Fuencarral-, Ciudad Universitaria -en Moncloa-, San Juan Bautista y Quintana -en Ciudad Lineal-, el Salvador -en San Blas-, Piovera -en Hortaleza - y Marroquina -en Moratalaz-. En general, se puede decir que el Madrid Interior y la Periferia Norte son el dominio de las clases altas y medias, mientras en la Periferia Sur dominan las clases bajas: éstas se hallan representadas fundamentalmente en los distritos de Latina, Carabanchel, Villaverde, Mediodia, Vallecas y Moratalaz, si bién, dentro de ellos, los barrios más próximos a la "almendra" suelen ser de clases medias.

El factor Envejecimiento Social, por su parte, se reúne con cargas positivas las variables relacionadas con los grupos demográficos de avanzada edad, además de la población llegada al municipio con anterio- 


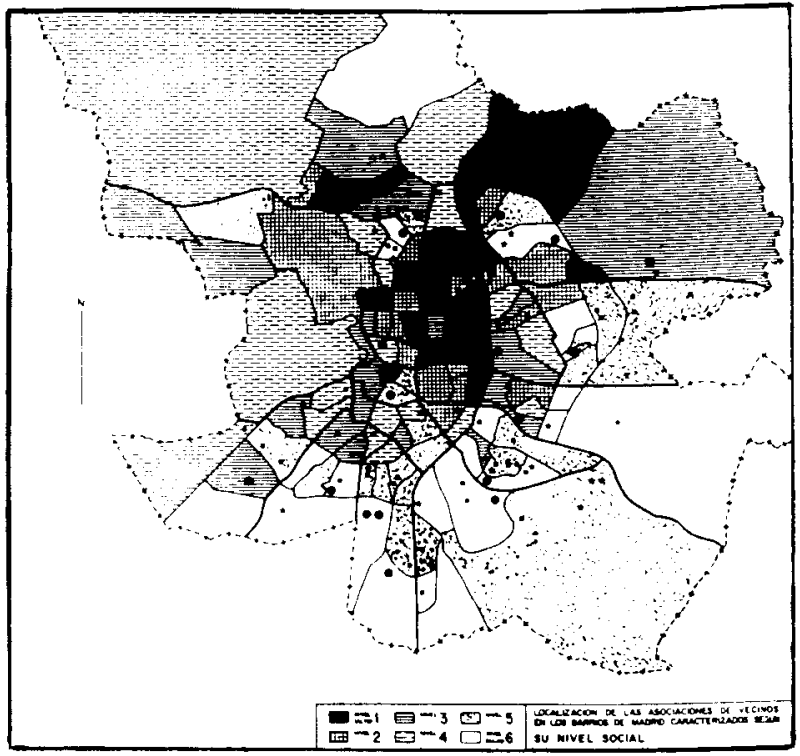

Fig. 8. Nivel social de los barrios de Madrid, según A. Castells (1981).

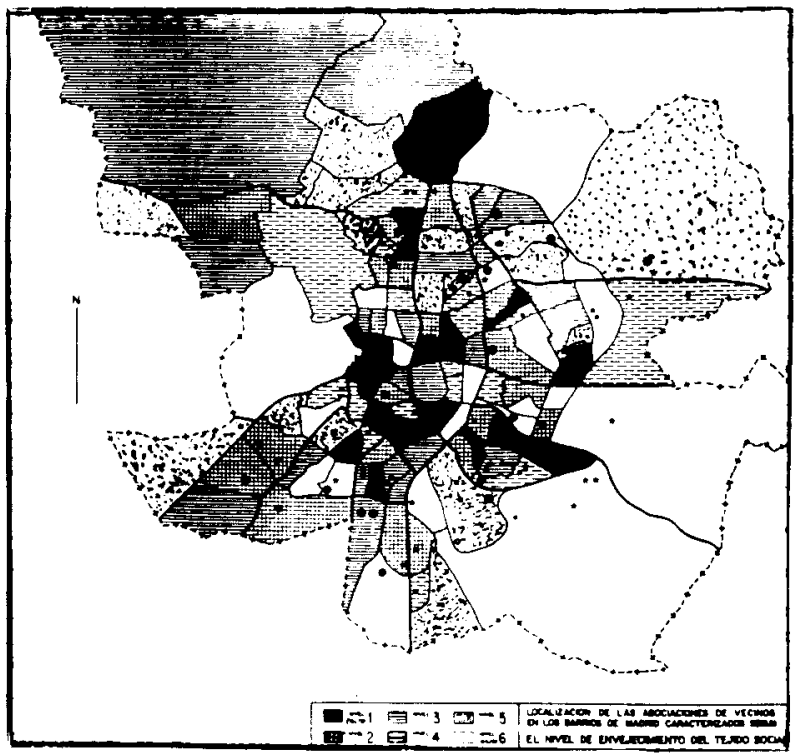

Fig. 9. Nivel de envejecimiento del tejido social en los barrios de Madrid, según A. Castells (1981). 
ridad a 1952, y los artesanos, comerciantes y trabajadores independientes, y con cargas negativas la población infantil y estudiantes y la comprendida entre 30 y 40 años. Como se aprecia, no es un factor puro. Su representación cartográfica no es excesivamente clara, como puede observarsen en la figura 9.

Del segundo factorial se deducen otros cuatro factores de tipo urbano, de los cuales el primero (Indicador de Espacio Residencial de Buena Calidad) es el más interesante y también el más claro, teniendo una estrecha relación en su distribución espacial con el factor Nivel Social del primer análisis: el espacio habitacional de mayor calidad se halla en el interior de la almendra (a excepción de los distritos de Centro y Arganzuela) en el Distrito de Moncloa, en algunos sectores de los distritos periféricos próximos a la "almendra" (Norte y Centro del de Ciudad Lineal y Oeste del de Moratalaz) y en varios barrios de la periferia más lejana de los distritos de Carabanchel y Latina. Los niveles más bajos se dan en algunos barrios del Centro y en los distritos de Vallecas, Mediodia y Villaverde.

Un segundo trabajo relacionado con la diferenciación residencial de Madrid, que utiliza técnicas de análisis multivariante, es el de A. del Campo Martín (1983), sobre las pautas espaciales de segregación social en la capital.

Partiendo de 14 variables sociodemográficas (extraídas de la Explotación Inicial del Padrón Municipal de habitantes de Madrid de 1977, públicada por COPLACO y de las publicaciones del Departamento de Estadística del Ayuntamiento de Madrid) correspondientes a los 119 barrios de Madrid que tenían población empadronada en la fecha, realiza un doble análisis: por una parte, una clasificación o agrupamiento de barrios sociodemográficamente homogéneos; y por otra, un análisis factorial "explicativo de los factores que determinan estas pautas de localización".

La primera tarea, esto es, la clasificación y ordenamiento de los barrios de Madrid, se realiza mediante un Análisis de Conglomerados o Cluster Analysis, técnica multivariante cuyo fin es la agrupación de individuos estadísticos u observaciones en conglomerados, racimos o amalgamientos, en relación con su proximidad, dentro de un espacio multidimensional, medida en función de unas dimensiones concretas, que son las variables analizadas para el conjunto de las observaciones. El análisis cluster referido a observaciones que tengan un contenido espacial, permite la delimitación de áreas homogéneas entre si y distantes de las demás, en función de las variables que se analicen, a través de los 
cluster o conglomerados que se formen con las unidades de observación.

A. del Campo, al realizar el análisis de conglomerados de los barrios de Madrid, en base a 14 variables sociodemográficas, escoge aquellos cluster que «mantienen una fuerte homogeneidad interna y cuya unión con otro grupo supone un gran salto en la escala de distancias $y$, por tanto, una pérdida sensible de información específica del grupo" (1983: 142); es decir, escoge agrupamientos "claramente significativos".

Las variables utilizadas, tanto para el análisis cluster, como para el análisis factorial, son las siguientes:

1. Estado Civil (\% casados);

2. Población Juvenil (\% menores de 14 años);

3. Población Senil (\% mayores de 65 años);

4. Sexo (\% varones);

5. Sirvientes (\% personal de servicio doméstico en el domicilio familiar);

6. Hacinamiento (población por viviendas residenciales);

7. Densidad Residencial (número de viviendas residenciales/Ha);

8. Bajo Nivel de Instrucción (\% población sin estudios, analfabeta o con primaria incompleta).

9. Alto Nivel de Instrucción (\% titulados superiores);

10. Mujeres Trabajadoras (\% mujeres trabajando fuera del hogar);

11. Cuadros Superiores (\% cuadros superiores);

12. Trabajadores Manuales (\% trabajadores manuales);

13. Densidad de Población (población por cada 10 Has.);

14. Viviencas con más de cinco miembros (\% viviendas con más de cinco miembros).

Del análisis se generan siete agrupamientos de barrios o «subáreas ecológicas", que aglutinan 86 de los 119 barrios que configuran la ciudad. El resto de las observaciones (33 barrios), bien se unen en distancias muy elevadas - lo que indica una fuerte especificidad del barrio-o bien se unen en distancias moderadas, pero al ser reducido su número, no aconseja la formación de subáreas: estos pequeños agrupamientos son denominados "áreas de segregación específica». 


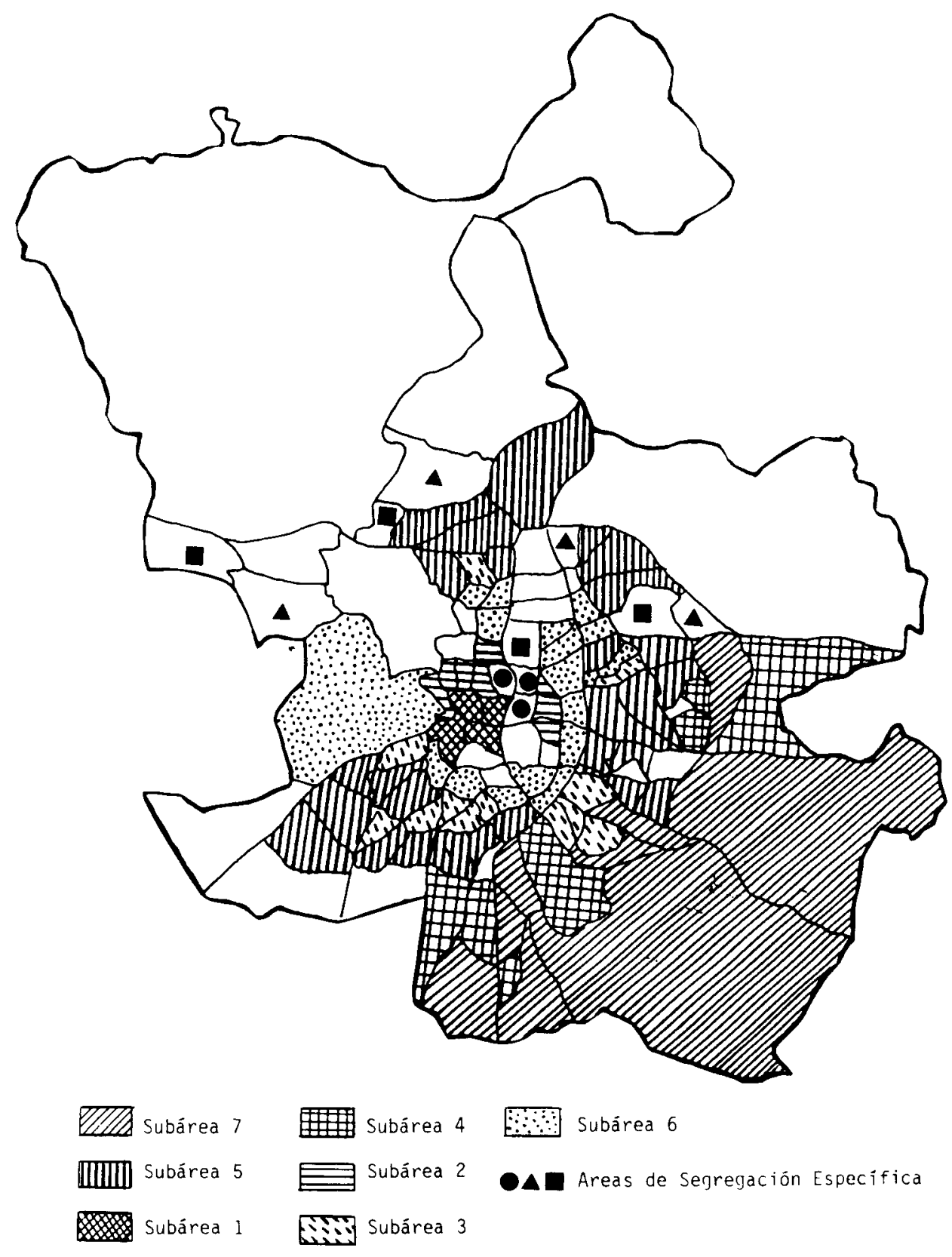

Fig. 10 Subáreas ecológicas de Madrid, según A. del Campo Martín (1983). (Elaboración propia a partir de los datos y mapas de A. del Campo Martín, 1983.) 
La figura 10 es una síntesis de los siete planos de subáreas que $A$. del Campo incluye en su artículo; en él también se reflejan las áreas de segregación específica a las que antes aludimos. En la simbología de nuesto mapa, las áreas aparecen por orden de mayor a menor homogeneidad interna, o, lo que es lo mismo, por orden de menor a mayor distancia dentro de la escala.

Las características generales de estas subáreas son:

- Subárea 1: engloba el 4 por 100 de la población del municipio. Su carácter más relevante es el fuerte envejecimiento de su población, tanto por cien de mujeres trabajadoras y escaso número de personas por vivienda. Agrupa cinco barrios del Distrito Centro.

- Subárea 2: comprende el 8,4 por 100 del total de población. Su característica fundamental es la alta densidad de viviendas residenciales y de población. Población envejecida (aunque no tanto como en la Subárea 1) y nivel de instrucción medio-alto. En general, clases sociales medias-altas. Todos los barrios de la subárea se localizan dentro de la "almendra" (Chamberí, Salamanca).

- Subárea 3: 13, 9 por 100 de la población. Área de bastante densidad residencial y de población. Alto porcentaje de casados. Escaso porcentaje de titulados y cuadros superiores. A. del Campo interpreta este agrupamiento como "una zona de transición en la que el grado de urbanización va descendiendo y la población se va ubicando en clases medias y medias-bajas". Los barrios del agrupamiento se localizan en la Periferia próxima, al sur de la "almendra».

- Subárea 4: 5,7 por 100 de la población. Alto porcentaje de trabajadores manuales; nivel de instrucción bajo; escaso porcentaje de cuadros superiores y alto porcentaje de viviendas con más de cinco miembros. Área de carácter obrero. Engloba barrios de la Periferia Sur y Este del municipio.

- Subárea 5: supone casi el 30 por 100 de la población de Madrid y tiene un elevado grado de homogeneidad. No tiene ninguna característica significativa: "se podría definir como el agrupamiento medio respecto del cual se segregan otros con caracteres especificos."

- Subárea 6: engloba el 12,6 por 100 de la población del municipio. Su homogeneidad interna es muy baja. Definida por el "elevado status social de su población". Tiene escaso nivel de viviendas con más de cinco miembros. Los barrios que forman este agrupamiento se localizan mayoritariamente dentro de la «almendra" y también, en algún caso, en la Periferia próxima. 
- Subárea 7: acumula el 8,4 por 100 de la población. Concentra un alto porcentaje de población con bajo nivel de instrucción y de trabajadores manuales. También se caracteriza por un escaso porcentaje de mujeres activas y carácter juvenil de su población. Todo ello configura una área obrera de reciente implantación. Se localiza en la Periferia Sureste de la ciudad.

Estas siete subáreas engloban el 83 por 100 de la población del municipio. El resto de la población forma parte, bien de áreas de segregación específica, bien de unidades espaciales de fuerte personalidad. Las áreas de segregación específica que señala $A$. del Campo son, por lo general, zonas de alto status social: tal es el caso de la configurada por los barrios de Castellana, Almagro y Recoletos, que se caracteriza también por un fuerte peso de la población femenina, de mujeres activas y el servicio doméstico, además de por tener una población envejecida. También de alto status es la agrupación de los barrios Fuentelarreina, Piovera, el Plantío y el Viso, que cuenta, asimismo, con un alto porcentaje de servicio doméstico y de viviendas con más de cinco miembros y baja densidad de población. De características parecidas a la anterior, el área de segregación específica de Mirasierra, Costillares, Aravaca y Palomas, se configura como residencia de población joven, de medio y alto status.

Para A. del Campo, el análisis cluster demuestra que en Madrid existen unas pautas espaciales en relación con la localización diferencial de la población; las características fundamentales son: la proximidad de barrios semejantes formando subáreas y el "carácter cuasi concéntrico (de las subáreas), lo que nos hace suponer la existencia de una serie de estrategias, respecto de una lucha por la localización en el espacio, en el cual la equidistancia del área central juega un importante papel" (1983: 148). Se refiere concretamente a la "coincidencia" en la disposición de las subáreas en círculos concéntricos, con el modelo de Burgess, coincidencia que, a nuestro juicio y a la vista del mapa, no resulta destacable.

La segunda parte del trabajo es la aplicación de las técnicas de análisis factorial de correspondencia a los 119 barrios de Madrid, en función de las 14 variables anteriormente relacionadas. Del proceso se extraen tres factores: Urbanización, Status Socioeconómico y Edad, que explican en conjunto el 93,65 por 100 de la varianza.

El factor Urbanización no tiene el mismo sentido aqui que en otros trabajos de Ecologia Factorial. En ellos se equipara a un eje «no familismo", mientras aqui tiene el sentido de "centralidad" o "residenciali- 
dad». El factor explica el 66,9 por 100 de la varianza, agrupando, con altas cargas positivas, las variables: densidad de población y densidad de viviendas y negativas: nivel de instrucción bajo y población juvenil.

El factor Status Socioeconómico (22,8 por 100 de la varianza), viene explicado por las variables nivel de instrucción alto, cuadros superiores y tanto por 100 de sirvientes, con cargas positivas, y los trabajadores manuales, con cargas negativas.

El factor Edad (3,93 por 100) reúne, con valores positivos, las variables población senil y mujeres en la fuerza de trabajo y con valores negativos, la población juvenil.

La posición de las variables y los barrios se representa, en función de sus coordenadas, en dos gráficos, de los cuales uno refleja los dos primeros factores y el otro los factores segundo y tercero. Resulta difícil extraer conclusiones sobre la diferenciación espacial de los barrios en función de los tres factores, ya que no se registran las puntuaciones factoriales de cada observación, ni se cartografían, lo cual habría resultado mucho más significativo y claro y habría completado el trabajo.

Las investigaciones de B. C. Jiménez Blasco (1984 y 1986) sobre la diferenciación residencal en Madrid, constituyen los trabajos más rigurosos publicados en la actualidad sobre el tema. De ellos, el primero (1984) es una aproximación metodológica, en la cual se realiza un Análisis Factorial de Componentes Principales de los 18 distritos y los 119 barrios de Madrid, en base a 16 variables sociodemográficas, extraídas de la explotación realizada por COPLACO de la actualización padronal de 1977.

Las variables de entrada, de las que la mitad son coincidentes con las incluidas por A. del Campo en su estudio sobre Madrid ya anteriormente comentado, son las siguientes: ción total;

1. Tanto por 100 de población de cinco a 19 años, sobre pobla-

2. Tanto por 100 de población de 65 años y más, sobre la población total;

3. Tanto por 100 de casados, sobre la población total;

4. Tanto por 100 de varones solteros, sobre la población total;

5. Tanto por 100 de mujeres dedicadas a sus labores, sobre la población total; total;

6. Tanto por 100 de trabajadores manuales, sobre la población 
7. Tanto por 100 de cuadros superiores de la producción, sobre la población total; total;

8. Tanto por 100 de menores y estudiantes, sobre la población

9. Tanto por 100 de titulados superiores, sobre la población total;

10. Tanto por 100 de viviendas habitadas por uno o dos miembros, sobre el total de viviendas;

11. Índice de hacinamiento: $n .^{\circ}$ de habitantes $/ n .{ }^{\circ}$ de viviendas;

12. Tanto por 100 de personas que trabajan en el servicio doméstico de las viviendas, sobre la población total;

13. Tanto por 100 de personas viudas, sobre la población total;

14. Tanto por 100 de cuadros medios, sobre la población total;

15. Tanto por 100 de personas con nivel de instrucción máximo, primaria incompleta, sobre la población total;

16. Tanto por 100 de población cuyo nivel de instrucción máximo es bachillerato superior, sobre la población total.

De la aplicación del A. F. de Componentes Principales, con rotación Varimax, se extraen dos factores de diferenciación residencial a nivel distrito, riue explican el 91,20 por 100 de la varianza: Nivel Socioeconómico y Urbanización, los mismos que resultan de la aplicación de la misma metodologia a los barrios de Madrid, donde, además, aparece un tercer factor de poca significación: entre los tres explican el 86,9 por 100 de la varianza. Tanto los coeficientes de correlación, como las saturaciones de cada factor y el tanto por 100 de varianza explicada, son superiores en el nivel distrito, hecho lógico, ya que la amplitud de las unidades de análisis encubre la heterogeneidad interna de cada una. Por ello, no nos pararemos a analizar los resultados a este nivel.

A nivel barrio, el primer factor extraído - Nivel Socioeconómicoexplica el 38,03 por 100 de la varianza y agrupa las variables: cuadros superiores, titulados superiores, nivel de bachillerato superior, servicio doméstico y cuadros medios, que se correlacionan positivamente entre si y negativamente con los trabajadores manuales y nivel primaria. En la representación cartográfica de las puntuaciones del factor, se tipifican seis umbrales: el superior está representado en barrios del interior (como Jerónimos, Recoletos, Castellana, el Viso, Nueva España y Vallehermoso) y algunos de la Periferia (Fuentelarreina, el Plantio, Ciudad Universitaria y Palomas, en los distritos de Moncloa, Fuencarral y Hortaleza). El se- 
gundo nivel también está representado fundamentalmente en el interior de la "almendra" y en algunos barrios de la Periferia próxima correspondientes a los distritos de Moncloa, Fuencarral, Ciudad Lineal y Hortaleza. En el otro extremo, en los dos últimos umbrales, los barrios con un nivel socioeconómico más bajo, se localizan, sobre todo, en la Periferia Suroriental del municipio, dentro de los distritos de Carabanchel, Villaverde, Mediodia, Vallecas, Moratalaz y San Blas. En líneas generales, el mapa resultante es parecido al de M. Castells (1981).

El segundo factor -Urbanización-explica el 31,97 por 100 de la varianza, agrupando, con altas correlaciones positivas, las variables: viudos, mayores de 65 años, viviendas con uno-dos ocupantes y varones solteros y negativas, las variables: menores y estudiantes y población de cinco a 19 años. Se trata del segundo eje diferenciador del modelo de Shevky y Bell. El factor adquiere su representación máxima en los barrios del Distrito Centro. En un segundo umbral se sitúan otros del interior de la "almendra" y algunos de la Periferia próxima (como Carolinas, en Villaverde y San Diego, Portazgo y Numancia, en Vallecas). En el otro extremo, con los valores más bajos del factor (dentro de los dos últimos umbrales) se registran la mayor parte de los barrios de la Periferia, caracterizados por un menor grado de consolidación urbana y mayor juventud de la población.

Por último, B. C. Jiménez Blasco realiza un análisis cluster de los barrios, introduciendo como variables las puntuaciones factoriales de los dos factores. De él se extraen tipos diferenciados de Áreas Sociales homogéneas, que se cartografian en el mapa de Madrid (fig. 11). El tipo A corresponde a los barrios con un nivel de urbanización bajo (familias jóvenes) y un nivel social medio-alto. El tipo $B$, a barrios también poco urbanizados y con status social más bajo que el tipo $A$. El tipo $C$ reúne barrios con puntuaciones bajas en ambos factores (ésto es, de bajo status socioeconómico y población joven). El tipo $D$ tiene bajo nivel de urbanización y nivel socioeconómico superior a los del tipo $\mathrm{C}$. El tipo $\mathrm{E}$ se caracteriza por agrupar barrios de bajo nivel social y un grado de urbanización medio. Por último, el grupo $\mathrm{F}$ corresponde a barrios con valores altos en ambos factores.

Mayor interés tiene un nuevo trabajo de B. C. Jiménez Blasco (1986), sobre la diferenciación residencial en Madrid, por realizar el estudio en tres niveles diferentes: distrito, barrio y sección censal, y utilizar diferentes métodos y técnicas de análisis para confirmar los resultados obtenidos. 
Una revisión de los estudios sobre diferenciación sociodemográfica, ...

TIPOLOGIA DE AREAS SOCIALES
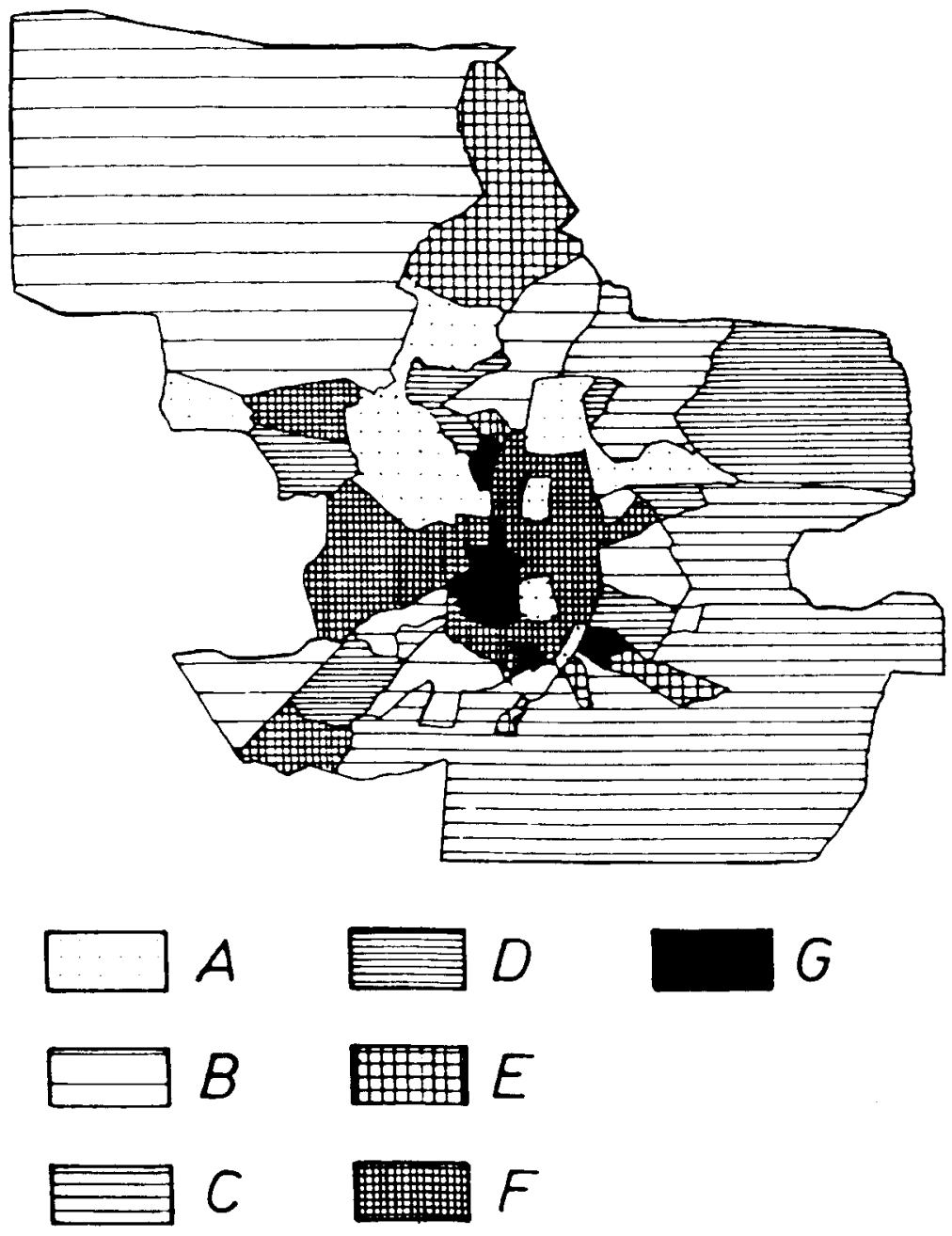

Delineación $V M$ dimenez glosso

Fig. 11. Tipología de áreas sociales en Madrid, según B. C. Jiménez Blasco (1984). 
Partiendo, como en el estudio anterior, de la explotación de la actualización padronal de 1977, llevada a cabo por COPLACO, selecciona 14 variables, de las cuales 13 son las mismas que introducía en el otro trabajo, incluyendo una nueva (Índice de Fertilidad) y eliminando tres (Varones Solteros de más de 15 años de edad, Índice de Hacinamiento y Personas Viudas). En base a ellas, ensaya distintos tipos de análisis multivariante, de cuyo resultado queda configurada la estructura de la diferenciación sociodemográfica de la ciudad.

El tamaño de las unidades de observación tiene una influencia decisiva en las soluciones factoriales en dos sentidos: en la progresiva complejidad de la estructura factorial a medida que el tamaño de la unidad de análisis disminuye, y también en la disminución de los valores de los coeficientes de correlación y las cargas de los factores, en la misma dirección. Así, a nivel distrito, la solución factorial es muy simple: dos factores - Rango Social y Envejecimiento- explican el 91,2 por 100 de la varianza total. El primero de ellos explica el 49,38 por 100 y el segundo el 41 ' 82 por 100 . La saturación de las variables incluidas en cada factor es más elevada por la mayor frecuencia de coeficientes de correlación significativos. No nos detendremos en este nivel por considerar las unidades de observación excesivamente amplias.

El análisis llevado a cabo a nivel de barrio municipal resulta más significativo. La estructura a este nivel se complica algo más, siendo las saturaciones de los factores más bajas. Con todo, los resultados obtenidos mediante el AF en Componentes Principales, son de indudable interés:

El primer factor -Rango social- explica el 38,64 por 100 de la varianza, incluyente las variables: cuadros superiores, titulados superiores, bachillerato superior, cuadros medios y servicio doméstico, con cargas positivas y los trabajadores manuales y nivel de primaria, con cargas negativas. Es un factor muy claro, que dentro del mapa madrileño muestra puntuaciones más elevadas en los barrios de la almendra (exceptuando los distritos de Centro y Arganzuela), en algunos barrios de los distritos de Fuencarral y Moncloa, en el Sector Norte de la Ciudad Lineal y en algunos del de Hortaleza. La puntuación más baja en el factor la alcanza el barrio de El Goloso (en Fuencarral), los de Hellín, Amposta y Arcos, en San Blas y San Cristóbal (en Mediodía), seguidos de gran parte de los barrios de la Periferia meridional y oriental de Madrid. (Fig. 12).

El segundo factor - Envejecimiento-Juventud- explica el 29,65 por 100 de la varianza y reúne las variables: población entre 5 y 19 años de 
Una revisión de los estudios sobre diferenciación sociodemográfica, ...

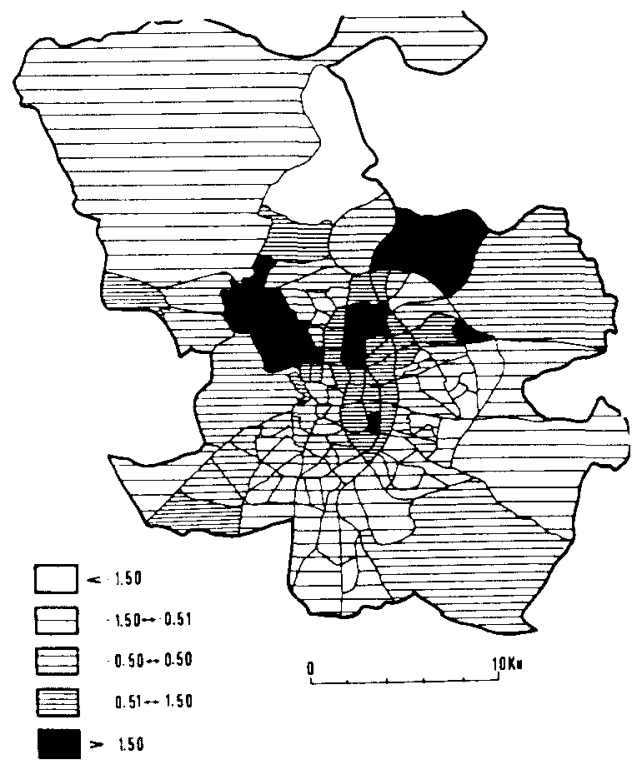

Fig. 12. Rango social de los barrios de Madrid (puntuaciones factoriales), según B. C. Jiménez Blasco (1986).

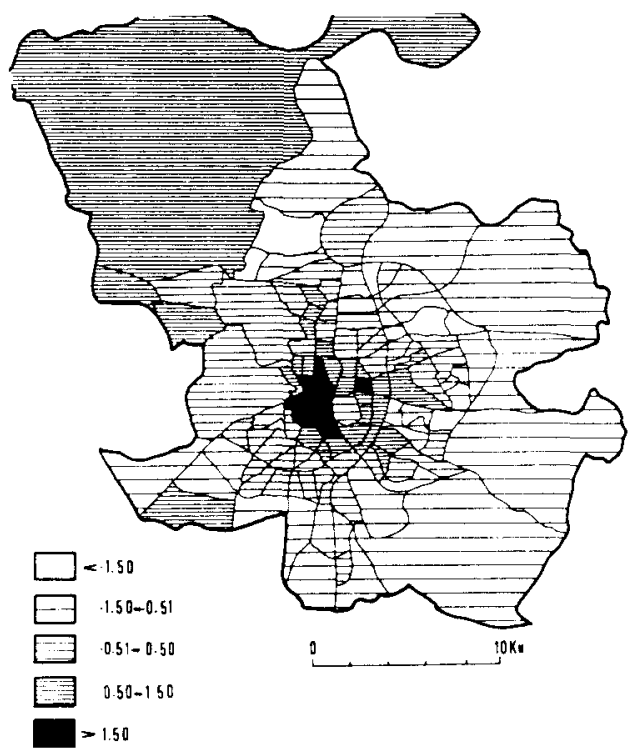

Fig. 13. Envejecimiento de los barrios de Madrid (puntuaciones factoriales según B. C. Jiménez Blasco (1986). 
edad, menores y estudiantes, $y$, en menor medida, el índice de fertilidad, todas ellas con valores positivos, y las variables: viviendas habitadas por uno o dos miembros y mayores de 65 años, con valores negativos. En la figura 13 se cartografían las puntuaciones factoriales. En general, son los barrios de la Periferia los más jóvenes y los de la "almendra", a excepción de los correspondientes al Distrito de Chamartín, los más envejecidos. Dentro de la Periferia existen, sin embargo, algunos barrios que muestran un elevado nivel de envejecimiento, como el Pardo (Fuencarral), el Plantío y Aravaca (Moncloa), Cuatro Vientos (Latina), Numancia y Olivar (Vallecas) y Ventas (Ciudad Lineal).

El tercer factor -Familismo- explica el 18,37 por 100 de la varianza e incluye las variables: personas casadas, índice de fertilidad, mujeres que se dedican a sus labores, con valores positivos y con valores negativos: las personas del servicio doméstico, la población de más de 65 años y titulados superiores (estas dos últimas variables, con valores muy bajos). Existe, por otra parte, una cierta relación entre los factores primero y tercero (Rango Social y Familismo), que muestran una baja correlación negativa $(-0,33)$. Mediante otros procedimiento ensayados, el factor Familismo pierde importancia, ganándola los otros dos, por lo que la autora no extrae las puntuaciones del factor, ni las cartografías.

Sin embargo, sí realiza un análisis cluster con las puntuaciones de los tres factores, a nivel barrio, deduciendo siete tipos de Áreas Sociales (cuyo grado de homogeneidad desconocemos) y cartografiando los resultados (fig. 14):

- Tipo 1: corresponde a barrios con una población muy envejecida y un nivel socioeconómico medio-bajo. Todos ellos se localizan en la "almendra" (todos los del Distrito Centro, más Delicias, Berruguete y Trafalfar), menos Numancia, que corresponde a Vallecas.

- Tipo 2: tiene puntuaciones altas en los tres factores, tratándose, "por lo general, de los llamados barrios burgueses", localizados sobre todo en la "almendra»: Pacífico, Estrella, Ibiza, Niño Jesis, Recoletos, Goya, Fuente del Berro, Guindalera, Lista, Castellana, Prosperidad, Ciudad Jardín, Hispanoamérica, Cuatro Caminos, Castillejos, Gaztambide, Arapiles, Almagro, Río Rosas, Casa de Campo, Argüelles, Valdemarín, Quintana y Concepción. Esta agrupación de barrios resulta, a primera vista, bastante heterogénea.

- Tipo 3: corresponde a barrios con población de bajo rango social y bastante elevado grado de envejecimiento (Imperial, Acacias, Chopera, Moguer, Valdeacederas. El Goloso, Cuatro Vientos, Carolinas, San Diego y Portazgo). 
Una revisión de los estudios sobre diferenciación sociodemográfica, ...

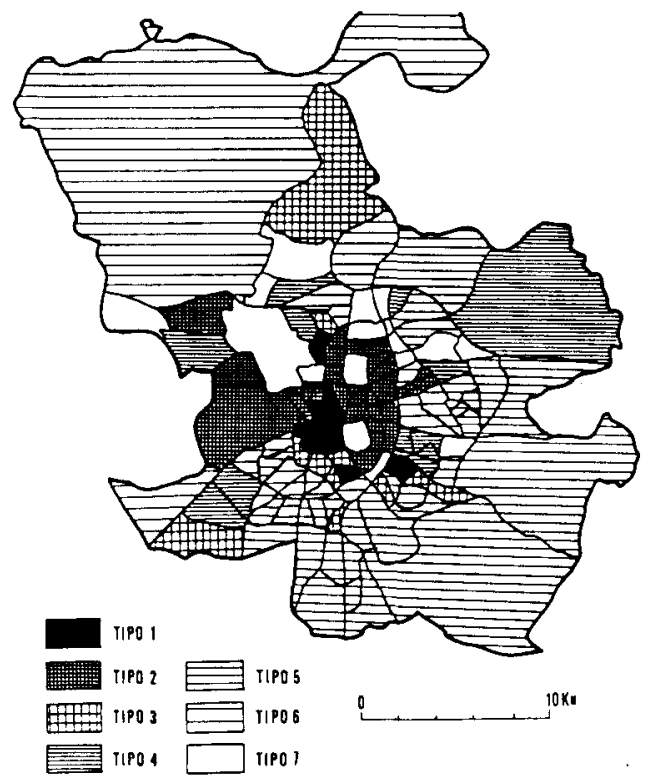

Fig. 14. Tipología de áreas sociales en la ciudad de Madrid, según B. C. Jiménez Blasco (1986).

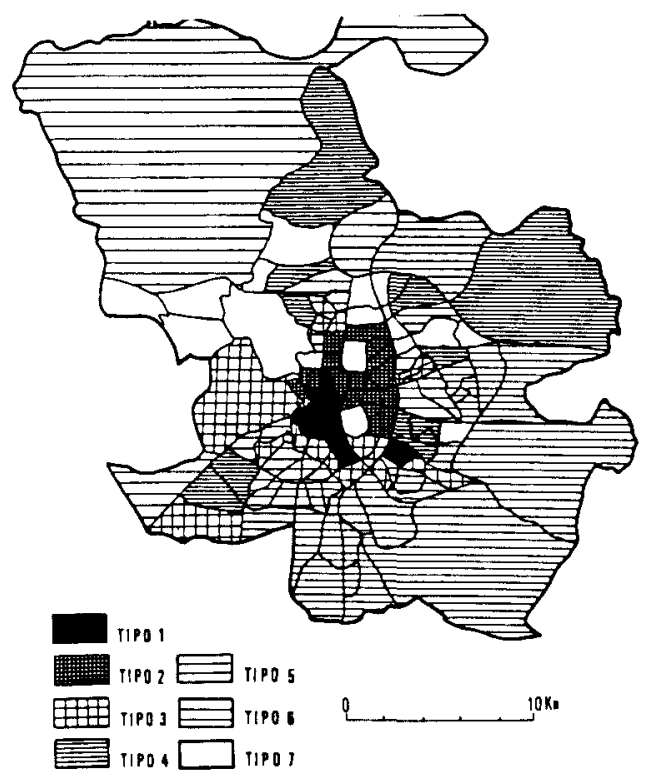

Fig. 15. Tipología de áreas sociales en la ciudad de Madrid, modificada por análisis discriminante, según B. C. Jiménez Blasco (1986). 
- Tipo 4: incluye barrios con valores medios en los factores. Se trata, en general, de barrios periféricos, aunque bastante urbanizados (Peñagrande, Valdezarza, Aravaca, Aluche, Águilas, Vinateros, Marroquina, Media Laguna, Fontarrón, Salvador, Barajas, Ápostol Santiago).

- Tipo 5: muestra un rango social bajo y una estructura demográfica joven. Está representando, sobre todo, en la Periferia Sur y Oriental (Cármenes, San Fermin, Simancas, Puerta Bonita, Buenavista, Pradolongo, Orcasitas, San Andrés, Los Angeles, Almendrales, Usera, Santa Catalina, los Rosales, San Cristóbal, Butarque, Villa de Vallecas, Picazo, Olivar, Palomeras, Pavones, Vicálvaro, Hellín, Amposta, Arcos, Rejas, Canillejas, Valdefuentes, Pinar del Rey, Canillejas).

- Tipo 6: incluye barrios con un mayor envejecimiento y también mayor rango social que el tipo 5 (Almenara, El Prado, El Pilar, Valverde, Puerta del Angel, Lucero, Campamento, Comillas, Opañel, San Isidro, Vista Alegre, Abrantes, Moscardó, Ventas, Pueblo Nuevo y San Pascual).

- Tipo 7: aglutina barrios con una escasa urbanización, rango social muy elevado y población en niveles medios de envejecimiento (Adelfas, Jerónimos, el Viso, Nueva España, Castilla, Vallehermoso, Fuentelarreina, Mirasierra, Ciudad Universitaria, El Plantío, San Juan Bautista, Colina, Atalaya, Costillares, Piovera y Palomas).

El tercer nivel de análisis empleado en el trabajo que estamos revisando es el de sección censal, nivel de desagregación utilizado, por otra parte, en multitud de trabajos de Ecología Factorial relativos a ciudades americanas, australianas y europeas. En el caso de Madrid, las unidades de observación son 2.228 y el análisis se realiza empleando 14 variables, de las que 12 son las mismas utilizadas en los análisis de los otros dos niveles y únicamente se sustituyen las variables índice de fertilidad y personas casadas, por varones solteros mayores de 15 años e índice de masculinidad. El método utilizado es el A.F. en Componentes Principales.

Las correlaciones entre variables a este nivel son más bajas que en los otros dos niveles; asimismo, la estructura factorial es más compleja y el porcentaje de varianza explicado, menor. De esta manera, se extraen cuatro factores, de los cuales únicamente los dos primeros son significativos, ya que el tercero aparece asociado a una sola variable (personas en el servicio doméstico) y el cuarto a dos (varones solteros de más de 15 años e índice de masculinidad). Estos dos últimos factores explican, respectivamente, el 14,07 por 100 y el 10,19 por 100 de la varianza. Por otra parte, los primeros factores son los mismos hallados en los otros niveles: Rango Social y Envejecimiento, que explican, entre ambos, el 62,72 por 100 de la varianza. 
El gran número de unidades de observación utilizadas no permitió realizar un análisis de conglomerados únicos para todas las secciones censales de Madrid, por falta de capacidad del programa de ordenador utilizado. Por ello, se realizaron a este nivel 18 análisis de conglomerados, uno por cada distrito, 10 cual hace que se pueda establecer una tipología de Áreas Sociales en base a la sección censal y a nivel de distrito, pero que estas áreas sociales no sean comparables de unos distritos a otros. Ello, unido a la consideración de la discutible homogeneidad y contenido propio de la sección censal como unidad espacial, resta interés al trabajo.

El estudio que resuminos -que constituye, sin lugar a dudas, un análisis serio y riguroso de la diferenciación residencial de la ciudad de Madrid, enfocada desde el punto de vista sociodemográfico- finaliza con un análisis de la varianza, en orden a establecer la adecuación de los factores de diferenciación sociodemográfica hallados, a los modelos clásicos de la estructura urbana, concluyendo que "la ciudad de Madrid se ajusta bastante bién al modelo de Murdie - el factor de rango social se estructura espacialmente de una forma sectorial y el factor envejecimiento o demográfico, según el modelo concéntrico»-, si bien en Madrid se da la peculiaridad de que el factor socioeconómico también se ajusta al modelo concéntrico, hecho que "puede entenderse en una ciudad como Madrid, donde vivir en el centro es un valor de prestigio" (1986: 611) y que se ha constatado también en otras ciudades mediterráneas.

\section{CONCLUSIÓN}

Dentro de los estudios revisados sobre la diferenciación sociodemográfica en la ciudad de Madrid, resultan de gran interés los que utilizan técnicas multivariantes, a pesar de que ninguno de ellos incluye variables relacionadas con la vivienda, aspecto que daría a estos trabajos un enfoque más amplio y una mayor perspectiva a la hora de conocer el espacio madrileño desde el punto de vista residencial.

Sobre este último grupo de trabajos, queremos poner de relieve to siguiente:

1. Llaman la atención las distintas conclusiones que se extraen a partir de los cuatro estudios analizados, a pesar de basarse todos ellos en la misma fuente y utilizar como unidad de análisis el barrio municipal. Ello pone de manifiesto la relación directa existente entre las variables 
de entrada y las soluciones factoriales, de manera que éstas vienen definidas antes por la matriz de datos inicial que por el Análisis Factorial.

2. Los factores diferenciadores más potentes en la distribución espacial de la población de Madrid son el status social y la edad, factores que ya habian sido puestos de relieve, aunque de forma intuitiva, en otros estudios clásicos.

3. El status socioeconómico o rango social se relaciona con variables muy concretas (cuadros superiores y medios y niveles de instrucción elevados, frente a niveles de instrucción bajos y trabajadores manuales). En la distribución espacial de los niveles de status, las localizaciones más claras corresponden a los grupos extremos del abanico: las clases altas por un lado, y las bajas por otro; existen, sin embargo, multitud de barrios que podríamos considerar genéricamente como "de clases medias", cuya tipificación no es tan clara, situándose en umbrales diferentes y a veces contrapuestos, en los distintos trabajos.

4. El segundo factor diferenciador en nuestra ciudad es la edad o el grado de envejecimiento-juventud, de la población. El factor tiene una estrecha relación con la antigüedad-modernidad de las áreas urbanas y también, a nuestro juicio, con el estadio dentro del ciclo familiar. Lo mismo que ocurría con el primer factor, las diferencias más claras se establecen entre áreas muy jóvenes y áreas muy envejecidas; los casos intermedios tienen diferentes tipificaciones en los distintos trabajos. Este factor, por otra parte, queda reflejado con mayor claridad y actualidad en los mapas confeccionados en base a los datos del Padrón Municipal de Habitantes de 1986, que se comentaron con anterioridad.

5. Existe un factor Familismo, detectado por B. C. Jiménez Blasco (1986) y contrapuesto al factor Urbanización (Jiménez Blasco, B. C., 1984), que no parece ser tan significativo de la diferenciación residencial, por no llegar a formar un factor potente. El familismo agrupa, con valores positivos, variables como personas casadas, tasa de fecundidad, mujeres dedicadas a sus labores, grupos de edades jóvenes, estudiantes y menores..., y con valores negativos, las variables: divorciados, viudos, población de más de 65 años, viviendas con uno o dos ocupantes..., que son características del factor Urbanización. Este factor, a nuestro juicio, se liga o se confunde en Madrid, a veces, con la factor envejecimientojuventud de la población y con el estadio en el ciclo familiar. Otras veces se halla ligeramente relacionado con el nivel socioeconómico (Jiménez Blasco, B. C., 1976). Algunas de las variables que lo componen son unidireccionales, alcanzando bajos índices de correlación, son poco significativas en el caso de Madrid, o pasan de uno a otro factor, según el 
método de rotación que se aplique a la matriz factorial y las variables que se hayan incluido en el estudio. En nuestro criterio, además, este factor diferenciador ha de cobrar mayor fuerza en nuevos ensayos realizados en base a padrones de habitantes más recientes en el tiempo.

Del análisis de los trabajos aquí expuestos se extrae como conclusión la existencia de cuatro tipos genéricos de áreas sociodemográficas, contrapuestas, en Madrid: a) áreas de alto status y población envejecida, cuya localización preferente es la "almendra", sobre todo el Ensanche y también barrios del Distrito Moncloa; b) áreas de alto status y población joven: nuevos barrios dentro de los distritos de Fuencarral, Moncloa - sobre todo en torno a la carretera de la Coruña-, Ciudad Lineal y Hortaleza, fundamentalmente; c) áreas de bajo status y población envejecida: localizadas en los distritos de Centro, Tetuán y Arganzuela, y también barrios surgidos de antiguos arrabales en torno a la ciudad central, como el de Ventas y algunos del Distrito de Vallecas, en la Periferia; d) áreas de bajo status y población joven: barrios localizados en la Periferia Sur y Sureste de la ciudad. Dentro de estos cuatro tipos genéricos de áreas, fruto de una simplificación de la realidad, existen multitud de tipos intermedios. 


\section{BIBLIOGRAFIA}

Abellán García, A., 1976: «Estructura por sexo y edad de los distritos de Madrid", Estudios Geográficos, núm. 144, Madrid, págs. 303-317.

Aguilera Arilla, M. J. y GonzÁlez Yanci, P., 1989: "Los contrastes del envejecimiento demográfico entre el municipio de Madrid y su comunidad autónoma", II Jornadas sobre Población Española, (Palma de Mallorca 1989. Universitat de les Isles Baleares. Palma de Mallorca, págs. 69-79.

AYUNTAMIENTO DE MADRID, 1982: Recuperar Madrid, Oficina Municipal del Plan.

AYUNTAMIENTO DE MADRID, 1983: “La Población en Madrid», Estudios Complementarios, Oficina Municipal del Plan.

Bodega Fernandez, M. I., et alli, 1982: “Evolución reciente y estructura de la población de Madrid y el SW de su municipio", Geographica,

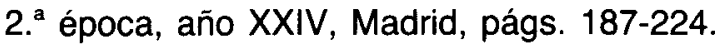

Campo Martín, A., 1983: “Una aplicación de la Ecología Factorial al estudio de pautas espaciales de segregación social en el municipio de Madrid", Ciudad y Territorio, núm. 57-58, Madrid, págs. 137-155.

CAStells, M., 1981: "La interacción entre crisis urbana y movimiento social urbano: la experiencia de Madrid", en ibidem, Crisis urbana $y$ cambio social. Madrid, Siglo XXI.

Chueca Goitia, G., 1974: "Sociología de Madrid", en ibidem, Madrid, ciudad con vocación de capital. Santiago de Compostela, Pico Sacro.

García Ballesteros, A. et alli, 1989: «El envejecimiento actual de la población madrileña: diferencias espaciales", II Jornadas sobre Po- 
Una revisión de los estudios sobre diferenciación sociodemográfica, ...

blación Española. (Palma de Mallorca 1989), Universitat de les Isles Baleares, Palma de Mallorca, págs. 207-227.

HUETZ DE LEMPS, A., 1972: "Madrid", en Les grandes villes du monde. Notes et études documentaires, París. La documentation française.

JIMÉNEZ BLASCO, B. C., 1984: "Aproximación metodológica al estudio de la diferenciación residencial urbana en Madrid", Anales de Geografía de la Universidad Complutense, núm. 4. Madrid, págs. 167-187.

JIMÉNEZ BLASCO, B. C., 1986: Estudio de la diferenciación residencial en la ciudad de Madrid. Tesis Doctoral. Facultad de Geografía e Historia, Universidad Complutense de Madrid.

OFICINA MUNICIPAL DEL PLAN, 1983: Documentos Sectoriales diversos, sobre Equipamiento. Madrid.

Timms, D., 1965: "Quantitative Techniques in Urban Social Geography», en CHORLEY, R. J. y HAGGeT, P., Frontiers in Geographical Teaching. London, págs.

Timms, D., 1976: “El mosaico urbano: hacia una teoría de la diferenciación residencial», Col. Nuevo Urbanismo, núm. 4. Madrid, IEAL. 